\title{
Seasonal breeding structure in house fly, Musca domestica L., populations
}

\author{
W. C. Black IV and E. S. Kiafsur
}

Department of Entomology, Iowa State University, Ames, Iowa 50011, U.S.A.

Allelic and genotypic frequencies were studied in four adult age groups of the common house fly. Electrophoresis was used to monitor allele frequencies at the $A d h$ locus and three $A m y$ loci at six farms on ten sampling dates from January to November 1983. No departures from random mating were noted. No differences in allele frequencies were found among sexes or age groups. Alleles at all loci showed seasonal trends. Frequency changes at Adh and Amyloci were large and occurred in parallel among all farms. Changes in the Amy + loci were small. At each locus, gene frequencies were statistically significantly different among farms, but differences were slight and not suggestive of strong local adaptation.

Allele frequencies were stable from autumn to spring in the overall population, but frequencies monitored at a single farm drifted significantly from estimates in the founding autumn subpopulation. In spring, allele frequencies were heterogeneous among farms due to genetic drift during winter. This differentiation diminished rapidly in June. Spatial genic differentiation was pronounced among flies 0-3 days old, but older flies were panmictic. Seasonal trends in gene frequencies among farms were similar in 1982 and 1983.

\section{INTRODUCTION}

The breeding structure of natural populations is a consequence of the mating patterns within and the amount of genetic flow among subpopulations. Field studies on brown snails (Helix aspersa, Selander and Kaufmann, 1975), Cepaea nemoralis (Johnson, 1976), and monarch butterflies (Daneus plexippus, Eanes and Koehn, 1978) demonstrated that random mating and rates of gene exchange are governed by the seasonal phenology of natural populations. These studies show that breeding structure can be dynamic in populations whose seasonal densities vary greatly.

We investigated how seasonal phenology influences breeding structure in the common house fly, Musca domestica. House flies are iteroparous, undergoing successive cycles of egg development and oviposition, the frequency of which is temperature dependent (Elvin and Krafsur, 1984). Studies were conducted in Ames, Iowa, where house flies overwinter as small, slowly breeding colonies in livestock facilities (Krafsur, 1985; Black and Krafsur, 1986a). In spring, these relic Economics Experiment Station, Ames, Iowa. Project No. 2411. colonies establish outdoor populations. In early spring, outdoor subpopulations consist predominantly of older, parous (i.e., having matured and laid at least one egg batch) adults that have clearly bred indoors. Populations increase rapidly in early summer, and continuous oscillations in age structure of adult populations occur during the breeding season; stable age distributions are not detected (Krafsur, 1985).

A first study on breeding structure was carried out in 1982 (Black and Krafsur, 1986b). We monitored spatial and temporal distributions of allelic and genotypic frequencies at six loci in the youngest age classes of adult female house flies. Allele frequencies changed significantly through time. Breeding structure changed seasonally in a manner suggesting midsummer convergence of subpopulations followed by a gradual divergence. The divergence of gene frequencies at farms began in mid-August when subpopulations were large and panmictic. Adult house flies are highly mobile (Bishopp, 1921), eliminating drift as an explanation of divergence. Strong local adaptation seemed a likely hypothesis.

A second study was conducted in 1983 to monitor seasonal differentiation in four age groups 
and both sexes of the house fly. Flies were sampled from January until November at six farms. Allele frequencies were recorded by age group to determine if the breeding structure observed among young adults in 1982 was repeated and if the same pattern appeared among older flies. A hypothesis of local adaptation among adult flies would be consistent with heterogeneity among all age groups. Alternatively, homogeneous gene frequencies among older flies and heterogeneous frequencies among the young flies would support a hypothesis of larval adaptation.

\section{METHODS}

\section{(i) Fly collections}

Adult house flies were collected with sweep nets from a beef farm, a swine farrowing facility, a dairy farm, and two pork confinement units (fig. 1). House fly larvae matured in the dung, spilled feed, and other organic material readily available

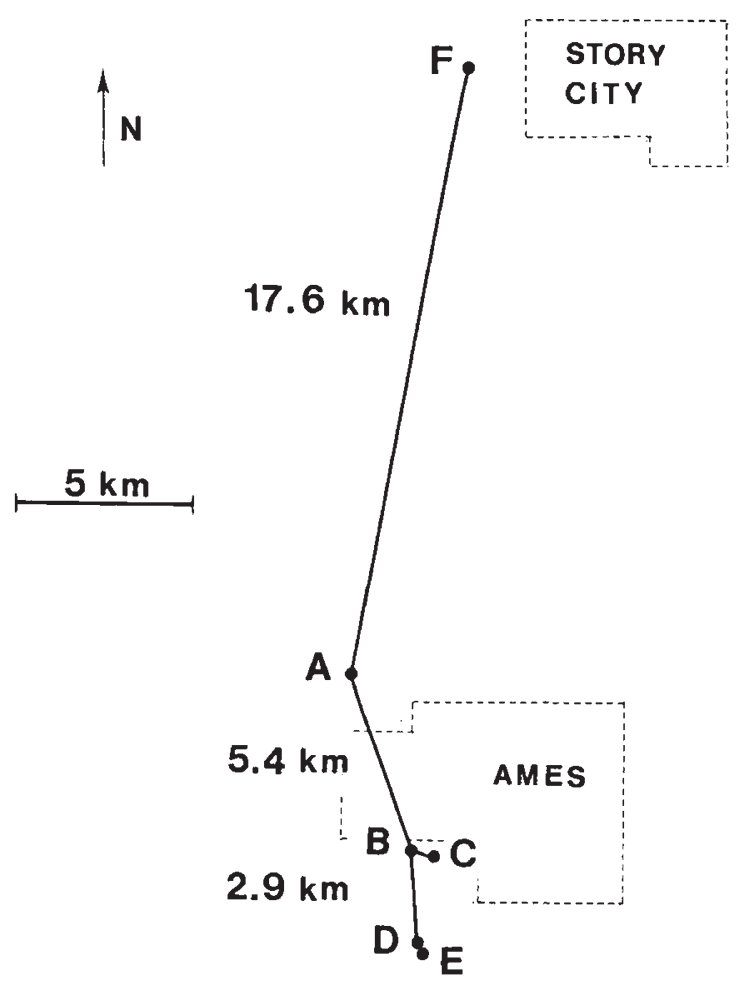

Figure 1 Map of the six sampling locations near Ames, Iowa. $A$, beef nutrition farm; b, swine farrowing sheds; C, dairy farm; D, sheep farm; E, pork production farm (south); F, pork production farm (north). at each farm. Rations fed to the animals differed among farms, raising the possibility of local adaptation. Flies were collected in winter from two heated buildings $30 \mathrm{~m}$ apart at the swine farrowing facility.

Flies were collected on 10 sampling occasions from late January until mid-November. Collections were made indoors in January, April and November. On each occasion, adults were placed in cages, returned alive to the laboratory, frozen and stored at $-70^{\circ} \mathrm{C}$.

\section{(ii) Age-grading}

Gonotrophic age in females was determined by examining the degree of ovarian development and the presence or absence of follicular relics. Age in males was determined by the presence or absence of pupal fat body. Four female and two male age groups were recognised.

Females of the youngest age class were recognised by the absence of yolk in their follicles. These females were zero to three days old at $21^{\circ} \mathrm{C}$ and termed "previtellogenic nullipars". The next oldest group consisted of females in which yolk occupied up to 66 per cent of the developing egg and whose ovaries showed no sign of an earlier oviposition. These females were $c a$. three to five days old and termed "vitellogenic nullipars". Ovarioles in the third age group exhibited signs of previous ovipositions. These "parous" females were at least six days old. The fourth age group were females in which yolk occupied 66 to 100 per cent of the developing egg. Follicle elongation prevented detection of previous ovipositions so that parity could not be accurately determined. These females were at least five days old.

\section{(iii) Electrophoretic procedures}

A random sample of $c a .100$ flies was taken from each collection. They were sexed, age-graded, transferred immediately to grinding buffer, homogenised and frozen at $-70^{\circ} \mathrm{C}$ awaiting electrophoresis. Electrophoretic procedures were as described (Black and Krafsur 1984; 1985a). Genotypes were scored at four loci: $A d h$ (alcohol dehydrogenase), Amy+ (fast) (anodally fast migrating amylase), Amy+ (slow), and Amy(fast). There were 21 alleles distributed among the six loci. Six alleles existed at the Amy+ (fast) locus, and five alleles occurred at each of the other loci. Electrophoretic assays were made of approximately 4300 flies representing 50 collections. 


\section{(iv) Analysis of data}

"Genestats" (Black and Krafsur, 1985b) was used to calculate allele frequencies and perform contingency chi-square tests (Workman and Niswander, 1970). Chi-square tests for departures from random mating and $F$-statistics were calculated following the methods of Weir and Cockerham (1985). "Linkdis" (Black and Krafsur, 1985c) was used to calculate linkage disequilibrium coefficients and check for significance.

Wright's (1978) hierarchical analysis of breeding structure for a subdivided population was used to identify sources of spatial differentiation in gene frequencies. In the analysis, sampling units are grouped into subpopulations according to their relative distances from one another. Farms (F) were the sampling units. They were grouped into subpopulations (S), which formed the total population $(T)$. The northern pork farm and the beef nutrition farm were treated as separate subpopulations, the swine farrowing facility and the dairy farm constituted a third subpopulation and the southern pork and sheep farms formed a fourth.

Three variance components were calculated. The variance in allele frequencies among farms $\left(F_{\mathrm{FT}}\right)$ is a function of the variance in allele frequencies among subpopulations $\left(F_{\mathrm{ST}}\right)$ and the variance in allele frequencies among adjacent farms in subpopulations $\left(F_{\mathrm{FS}}\right)$. The three statistics are related by the equation,

$$
F_{\mathrm{FT}}=F_{\mathrm{ST}}+F_{\mathrm{FS}}-\left(F_{\mathrm{ST}} \times F_{\mathrm{FS}}\right)
$$

Where flies are completely panmicitic, all $F$-statistics are zero. When flies produced at farms are differentiated by selection or genetic drift, then allele frequencies within the same subpopulation will be heterogeneous and $F_{\mathrm{FS}} \geqq F_{\mathrm{ST}}$. If flies produced within subpopulations are panmictic and subpopulations are differentiated because of distance, local selection pressures, barriers to mating, etc., then $F_{\mathrm{ST}} \leqq F_{\mathrm{FS}}$.

Correlation coefficients between allele frequencies were converted to a normalised scale with Fisher's $z$-transformation. Chi-square tests for the homogeneity of $z$-values were computed following the procedure in Sokal and Rohlf (1969). A mean $z$-value of all possible correlations was calculated and back-transformed to estimate a common correlation.

\section{RESULTS}

\section{(i) Temporal and spatial variation in age structure}

The proportions of previtellogenic and parous flies are listed by sampling dates (table 1 ) and by farms (table 2). The proportions of parous flies were heterogeneous among farms during May and June, converged in July, and remained homogeneous until November. The proportions of previtellogenic flies were homogeneous early in the summer, became heterogeneous in July, and remained so throughout late summer and autumn. The data indicate that age structures fluctuated throughout the season and stable age distributions were not obtained. The seasonal mean PP was homogeneous among farms in summer and autumn, suggesting

Table 1 Proportions of previtellogenic (PPV) and parous (PP) flies according to sampling dates. Chi-square statistics test for homogeneity among six farms ( 5 d.f.)

\begin{tabular}{lrrrr}
\hline Date & \multicolumn{1}{l}{ PPV } & \multicolumn{1}{c}{$\chi^{2}$} & \multicolumn{1}{c}{ PP } & \multicolumn{1}{c}{$\chi^{2}$} \\
\hline 23 May & $32 / 80=40 \cdot 0 \%$ & $6 \cdot 4$ & $33 / 48=68 \cdot 8 \%$ & $13 \cdot 3 \dagger$ \\
6 June & $43 / 98=43 \cdot 9 \%$ & $5 \cdot 1$ & $34 / 55=61 \cdot 8 \%$ & $10 \cdot 4^{*}$ \\
20 June & $29 / 113=25 \cdot 7 \%$ & $3 \cdot 7$ & $53 / 83=62 \cdot 7 \%$ & $18 \cdot 3 \dagger$ \\
11 July & $70 / 201=34 \cdot 8 \%$ & $30 \cdot 7 \ddagger$ & $97 / 131=74 \cdot 1 \%$ & $10 \cdot 9$ \\
1 August & $35 / 222=15 \cdot 8 \%$ & $13 \cdot 8 *$ & $113 / 187=60 \cdot 4 \%$ & $2 \cdot 2$ \\
12 September & $81 / 231=35 \cdot 1 \%$ & $12 \cdot 6 *$ & $62 / 150=41 \cdot 3 \%$ & $7 \cdot 1$ \\
10 October & $40 / 160=25 \cdot 0 \%$ & $10 \cdot 3$ & $29 / 120=24 \cdot 2 \%$ & $8 \cdot 0$ \\
6 November & $84 / 170=49 \cdot 4 \%$ & $63 \cdot 6 \ddagger$ & $28 / 86=32 \cdot 6 \%$ & $12 \cdot 6 \dagger$ \\
Total & $414 / 1275=32 \cdot 5 \%$ & & $448 / 860=52 \cdot 1 \%$ & \\
\hline
\end{tabular}

\footnotetext{
* $P \leqq 0.05$

$\dagger P \leqq 0.01$

$\ddagger P \leqq 0.001$
}

Tests for seasonal homogeneity in PPV and PP:

PPV $\chi^{2}(7$ d.f. $)=66 \cdot 1 \ddagger$

PP $\quad \chi^{2}(7$ d.f. $)=99 \cdot 2 \ddagger$ 
Table 2 Proportions of previtellogenic (PPV) and parous (PP) flies according to sampling location. Chi-square statistics test for homogeneity among eight sampling dates ( $7 \mathrm{~d} . \mathrm{f}$.)

\begin{tabular}{llcll}
\hline Farm & PPV & $\chi^{2}$ & PP & $\chi^{2}$ \\
\hline Pork (north) & $76 / 176=43 \cdot 2 \%$ & $67 \cdot 6 \ddagger$ & $58 / 100=58 \cdot 0 \%$ & $16 \cdot 5 \dagger$ \\
Nutrition & $41 / 208=19 \cdot 7 \%$ & $8 \cdot 0$ & $82 / 167=49 \cdot 1 \%$ & $40 \cdot 1 \ddagger$ \\
Swine & $85 / 284=30 \cdot 0 \%$ & $37 \cdot 1 \ddagger$ & $86 / 199=43 \cdot 2 \%$ & $41 \cdot 4 \ddagger$ \\
Dairy & $70 / 215=32 \cdot 5 \%$ & $31 \cdot 0 \ddagger$ & $74 / 145=51 \cdot 0 \%$ & $14 \cdot 9 *$ \\
Sheep & $78 / 186=41 \cdot 9 \%$ & $25 \cdot 1 \ddagger$ & $65 / 108=60 \cdot 2 \%$ & $23 \cdot 1 \ddagger$ \\
Pork (south) & $64 / 206=31 \cdot 1 \%$ & $7 \cdot 4$ & $83 / 141=58 \cdot 9 \%$ & $24 \cdot 9 \ddagger$ \\
\hline
\end{tabular}

$* P \leqq 0.05$

$\dagger P \leqq 0.01$

$\ddagger P \leqq 0.001$

Tests for spatial homogeneity in PPV and PP:

PPV $\chi^{2}(5$ d.f. $)=33 \cdot 3 \ddagger$

PP $\quad \chi^{2}(5$ d.f. $)=13 \cdot 8^{*}$

similar survival probabilities (Krafsur, 1985). The heterogeneity in PP early in the season can probably be attributed to inadequate sampling frequency.

The greatest PPV were consistently found at the sheep farm and the northern pork farm. The smallest PPV were consistently noted at the beef operation. At the sheep and pork farms, flies were swept from walls adjacent to spilled feed, manure, and moist straw in which larvae develop. At the beef farm, flies were collected indoors where there was no immediately local breeding. The proportions of previtellogenic females were directly related to the proximity of collections to larval breeding sites. These results are consistent with earlier observations in 1982 (Black and Krafsur, 1986b).

\section{(ii) Allele frequencies between sexes and among age groups}

Chi-square tests of homogeneity in allele frequencies between sexes were performed for each farm and sampling date. Of 179 tests, $12(6.7$ per cent $)$ were significant at the 5 per cent level. This was consistent with expectations for a type I error. Significant values were evenly distributed among farms, loci, and dates.

Tests of homogeneity between sexes of the same age were performed to remove possible confounding age effects. The proportion of significant tests $(8 / 180=0 \cdot 044)$ was consistent with expectation. No real differences in allele frequencies between sexes were detected. Therefore, gene frequencies of teneral males were combined with those of teneral and nulliparous females. These are henceforward referred to as "young" flies.
Allele frequencies in young and parous adults were examined by farm and sampling dates. Of 162 chi-square tests, only $5 \cdot 5$ per cent were statistically significant, and these were independently distributed among farms, dates, and loci.

\section{(iii) Genotypic frequencies}

The goodness of fit of observed genotypic frequencies to those expected under random mating was tested. Eighteen of $184(9.8$ per cent) tests were significant. This was more than the 5 per cent expected for a type I error $\left(\chi^{2}=8.86\right.$ [1 d.f.], $P=$ $0 \cdot 003)$. Significant departures from expectation were independently distributed among loci, dates, and farms. Sixteen of the significant deviations from Hardy-Weinberg expectations involved heterozygote deficiencies. This suggested the hypothesis that the deviations resulted from slight differences among age groups and sexes according to Wahlund's principle (Hartl, 1980). Therefore, random mating was tested in age groups. The number of significant tests decreased to nine of 184 ( 4.9 per cent) in "young" flies and to 10 of 155 (6.5 per cent) among parous females. When random mating was examined by sex, 4.9 per cent of tests were significant in males and 6.5 per cent in females. These statistical results show that slight differences in allele frequencies in age and sex created the excess of homozygotes.

\section{(iv) Temporal trends in allele frequencies}

The frequencies and standard errors of the most common alleles are set forth in table 3 . Correlations were calculated between allele frequencies for all pairs of farms (table 4) to determine if alleles followed similar seasonel patterns. 
Gene frequencies at the $A m y-$ and $A d h$ loci were significantly different among dates at each farm. The seasonal maximum and minimum differed by 18 per cent. Adh 2 declined in midsummer and increased in autumn. Amy -2 increased in midsummer and decreased in autumn. Correlation coefficients for the $A d h$ and $A m y$ - loci were consistently positive, and common correlations were large. Trends in gene frequencies occurred in parallel among farms.

\section{(v) Linkage disequilibrium}

Linkage disequilibrium coefficients for locus pairs were calculated and tested for significance. Of 276 independent tests 28 were statistically significant. This was greater than expected by chance alone $\left(\chi^{2}[1\right.$ d.f. $\left.]=15 \cdot 4, \quad P \leqq 0 \cdot 0001\right)$. Only nine significant results involved common alleles and this was consistent with expectations. Significant values were homogeneously distributed among farms, loci and dates. There was no discernable pattern to the linkage disequilibrium. The same result was noted when analyses were performed on flies of the youngest age class.

\section{(vi) Overwintering}

Allele frequencies were homogeneous between sheds and did not change from January through April (table 5). Gene frequencies in October 1982 and January 1983 were homogeneous but became significantly different between October and April $\left(\chi^{2}[8\right.$ d.f.] $=28.63, P=0.0004)$ and between April and May $\left(\chi^{2}[12\right.$ d.f. $\left.]=33.98, P=0.001\right)$. Allele frequencies at the swine farm changed significantly during winter (October vs. May: $\chi^{2}[9$ d.f. $]=33 \cdot 59$, $P=0.0001$ ), but allele frequencies averaged over all farms in October were homogeneous with average frequencies at farms in May.

\section{(vii) Breeding structure}

$F$-statistics are plotted for all fly ages (fig. 2), parous flies (fig. 3) and "young" flies (fig. 4). Gene frequencies were heterogeneous among farms in May (fig. 2). Heterogeneity rapidly declined in June, frequencies became homogeneous in July and remained so until November. $F_{F S}$ contributed most to $F_{\mathrm{Fr}}$ in spring, showing that most of the differentiation among farms was due to drift. $F_{\mathrm{FS}}$ declined rapidly in spring, reached zero by $20 \mathrm{June}$, and remained small during the rest of the season.

Seasonal trends in $F_{\mathrm{Fr}}$ in flies of all ages were correlated with trends in $F_{\mathrm{FT}}$ in parous flies (fig. 3 ). This suggests that breeding structure of parous adults was similar to the breeding structure of the general adult population. Furthermore, excluding November, $F_{\mathrm{Fr}}$ and $F_{\mathrm{FS}}$ were congruent among all adults (fig. 2) and parous adults (fig. 3).

Seasonal trends in $F_{\mathrm{FT}}$ in parous and "young" adults (fig. 4) were independent, and this $F$ statistic varied erratically among the youngest age group. In May and June, $F_{\mathrm{Fr}}$ and $F_{\mathrm{FS}}$ were independent among "young" flies. From August through November, $F_{\mathrm{FS}}$ increased with $F_{\mathrm{FT}}$ indicating that much of the variation in allele frequencies among all farms was contributed by variation among adjacent farms. Local adaptation is an attractive possibility for this differentiation.

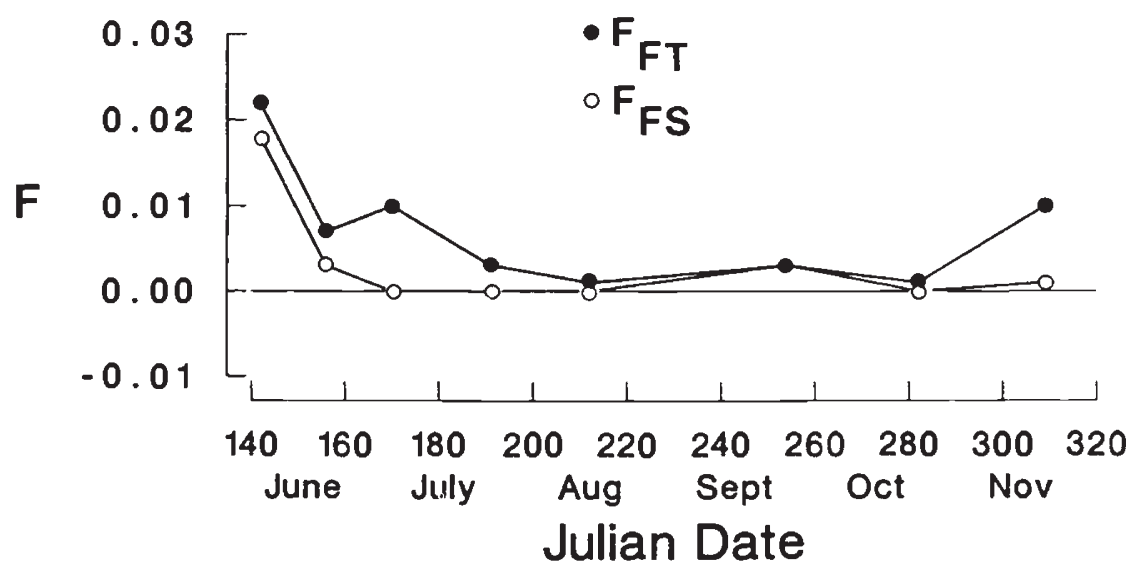

Figure 2 Seasonal fluctuations in Wright's $F_{\mathrm{FT}}$ and $F_{\mathrm{FS}}$ among all flies. 
Table 3 Frequencies and standard errors (in parentheses) of the most common allele at four enzymatic loci in the house fly in 1983

\begin{tabular}{|c|c|c|c|c|c|c|c|}
\hline \multirow[b]{2}{*}{ Date } & \multicolumn{6}{|c|}{ Farm } & \multirow[b]{2}{*}{ Population } \\
\hline & $\begin{array}{l}\text { Pork } \\
\text { (North) }\end{array}$ & Nutr. & Swine & Dairy & Sheep & $\begin{array}{l}\text { Pork } \\
\text { (South) }\end{array}$ & \\
\hline \multicolumn{8}{|c|}{ Adh 2} \\
\hline 23 May & $\begin{array}{c}0.625 \\
(0.070)\end{array}$ & - & $\begin{array}{c}0.772 \\
(0.032)\end{array}$ & $\begin{array}{c}0.661 \\
(0.035)\end{array}$ & - & $\begin{array}{c}0.745 \\
(0.031)\end{array}$ & $\begin{array}{c}0.704 \\
(0.018)\end{array}$ \\
\hline 6 June & $\begin{array}{l}0.759 \\
(0.041)\end{array}$ & - & $\begin{array}{c}0.760 \\
(0.030)\end{array}$ & $\begin{array}{c}0.705 \\
(0.040)\end{array}$ & $\begin{array}{c}0.675 \\
(0.033)\end{array}$ & $\begin{array}{c}0.725 \\
(0.032)\end{array}$ & $\begin{array}{c}0.723 \\
(0.015)\end{array}$ \\
\hline 20 June & $\begin{array}{l}0.750 \\
(0.050)\end{array}$ & $\begin{array}{c}0.700 \\
(0.032)\end{array}$ & $\begin{array}{c}0.609 \\
(0.035)\end{array}$ & $\begin{array}{c}0.680 \\
(0.033)\end{array}$ & $\begin{array}{c}0.645 \\
(0.034)\end{array}$ & $\begin{array}{c}0.697 \\
(0.033)\end{array}$ & $\begin{array}{c}0.673 \\
(0.014)\end{array}$ \\
\hline 11 July & $\begin{array}{l}0.611 \\
(0.035)\end{array}$ & $\begin{array}{c}0.587 \\
(0.035)\end{array}$ & $\begin{array}{c}0.462 \\
(0.037)\end{array}$ & $\begin{array}{c}0.554 \\
(0.039)\end{array}$ & $\begin{array}{c}0.512 \\
(0.038)\end{array}$ & $\begin{array}{c}0.586 \\
(0.036)\end{array}$ & $\begin{array}{c}0.553 \\
(0.015)\end{array}$ \\
\hline 1 August & $\begin{array}{l}0.602 \\
(0.035)\end{array}$ & $\begin{array}{c}0.580 \\
(0.035)\end{array}$ & $\begin{array}{c}0.591 \\
(0.036)\end{array}$ & $\begin{array}{c}0.560 \\
(0.035)\end{array}$ & $\begin{array}{c}0.710 \\
(0.034)\end{array}$ & $\begin{array}{c}0.582 \\
(0.035)\end{array}$ & $\begin{array}{c}0.602 \\
(0.014)\end{array}$ \\
\hline 12 September & $\begin{array}{l}0.590 \\
(0.036)\end{array}$ & $\begin{array}{c}0.480 \\
(0.036)\end{array}$ & $\begin{array}{c}0.561 \\
(0.035)\end{array}$ & $\begin{array}{c}0.535 \\
(0.038)\end{array}$ & $\begin{array}{c}0.478 \\
(0.037)\end{array}$ & $\begin{array}{c}0.617 \\
(0.035)\end{array}$ & $\begin{array}{c}0.544 \\
(0.015)\end{array}$ \\
\hline 10 October & $\begin{array}{l}0.660 \\
(0.035)\end{array}$ & $\begin{array}{l}0.594 \\
(0.035)\end{array}$ & $\begin{array}{c}0.528 \\
(0.037)\end{array}$ & $\begin{array}{c}0.600 \\
(0.039)\end{array}$ & $\begin{array}{c}0.647 \\
(0.037)\end{array}$ & $\begin{array}{c}0.625 \\
(0.035)\end{array}$ & $\begin{array}{c}0.609 \\
(0.015)\end{array}$ \\
\hline 7 November & $\begin{array}{l}0.619 \\
(0.045)\end{array}$ & $\begin{array}{c}0.558 \\
(0.049)\end{array}$ & $\begin{array}{c}0.633 \\
(0.035)\end{array}$ & $\begin{array}{c}0.682 \\
(0.036)\end{array}$ & $\begin{array}{c}0.682 \\
(0.070)\end{array}$ & $\begin{array}{c}0.563 \\
(0.037)\end{array}$ & $\begin{array}{c}0.643 \\
(0.015)\end{array}$ \\
\hline Mean & $\begin{array}{l}0.639 \\
(0.014)\end{array}$ & $\begin{array}{c}0.585 \\
(0.015)\end{array}$ & $\begin{array}{c}0.611 \\
(0.012)\end{array}$ & $\begin{array}{c}0.621 \\
(0.013)\end{array}$ & $\begin{array}{c}0.615 \\
(0.014)\end{array}$ & $\begin{array}{r}0.645 \\
(0.12)\end{array}$ & $\begin{array}{c}0.623 \\
(0.005)\end{array}$ \\
\hline \multicolumn{8}{|c|}{$A m y+($ fast $) 4$} \\
\hline 23 May & $\begin{array}{l}0.750 \\
(0.063)\end{array}$ & - & $\begin{array}{c}0.641 \\
(0.034)\end{array}$ & $\begin{array}{c}0.833 \\
(0.027)\end{array}$ & - & $\begin{array}{c}0.740 \\
(0.031)\end{array}$ & $\begin{array}{c}0.737 \\
(0.018)\end{array}$ \\
\hline 6 June & $\begin{array}{l}0.694 \\
(0.044)\end{array}$ & - & $\begin{array}{c}0.781 \\
(0.030)\end{array}$ & $\begin{array}{c}0.746 \\
(0.038)\end{array}$ & $\begin{array}{c}0.740 \\
(0.031)\end{array}$ & $\begin{array}{c}0.677 \\
(0.033)\end{array}$ & $\begin{array}{c}0.730 \\
(0.015)\end{array}$ \\
\hline 20 June & $\begin{array}{l}0.750 \\
(0.050)\end{array}$ & $\begin{array}{c}0.758 \\
(0.030)\end{array}$ & $\begin{array}{c}0.763 \\
(0.030)\end{array}$ & $\begin{array}{c}0.727 \\
(0.032)\end{array}$ & $\begin{array}{c}0.795 \\
(0.029)\end{array}$ & $\begin{array}{c}0.776 \\
(0.030)\end{array}$ & $\begin{array}{c}0.763 \\
(0.013)\end{array}$ \\
\hline 11 July & $\begin{array}{l}0.734 \\
(0.032)\end{array}$ & $\begin{array}{c}0.813 \\
(0.028)\end{array}$ & $\begin{array}{c}0.796 \\
(0.029)\end{array}$ & $\begin{array}{c}0.755 \\
(0.031)\end{array}$ & $\begin{array}{c}0.758 \\
(0.031)\end{array}$ & $\begin{array}{c}0.750 \\
(0.032)\end{array}$ & $\begin{array}{c}0.768 \\
(0.012)\end{array}$ \\
\hline 1 August & $\begin{array}{l}0.815 \\
(0.027)\end{array}$ & $\begin{array}{c}0.732 \\
(0.031)\end{array}$ & $\begin{array}{c}0.783 \\
(0.029)\end{array}$ & $\begin{array}{c}0.776 \\
(0.030)\end{array}$ & $\begin{array}{c}0.740 \\
(0.031)\end{array}$ & $\begin{array}{c}0.760 \\
(0.31)\end{array}$ & $\begin{array}{c}0.768 \\
(0.012)\end{array}$ \\
\hline 12 September & $\begin{array}{l}0.760 \\
(0.031)\end{array}$ & $\begin{array}{c}0.724 \\
(0.032)\end{array}$ & $\begin{array}{c}0.720 \\
(0.032)\end{array}$ & $\begin{array}{c}0.805 \\
(0.030)\end{array}$ & $\begin{array}{c}0.716 \\
(0.032)\end{array}$ & $\begin{array}{c}0.796 \\
(0.041)\end{array}$ & $\begin{array}{c}0.749 \\
(0.013)\end{array}$ \\
\hline 10 October & $\begin{array}{l}0.801 \\
(0.029)\end{array}$ & $\begin{array}{c}0.795 \\
(0.029)\end{array}$ & $\begin{array}{c}0.705 \\
(0.032)\end{array}$ & $\begin{array}{c}0.775 \\
(0.031)\end{array}$ & $\begin{array}{c}0.804 \\
(0.029)\end{array}$ & $\begin{array}{c}0.823 \\
(0.028)\end{array}$ & $\begin{array}{c}0.783 \\
(0.012)\end{array}$ \\
\hline 7 November & $\begin{array}{l}0.809 \\
(0.037)\end{array}$ & $\begin{array}{c}0.764 \\
(0.041)\end{array}$ & $\begin{array}{c}0.790 \\
(0.029)\end{array}$ & $\begin{array}{c}0.832 \\
(0.027)\end{array}$ & $\begin{array}{c}0.727 \\
(0.067)\end{array}$ & $\begin{array}{c}0.761 \\
(0.032)\end{array}$ & $\begin{array}{c}0.792 \\
(0.013)\end{array}$ \\
\hline Mean & $\begin{array}{l}0.770 \\
(0.013)\end{array}$ & $\begin{array}{c}0.764 \\
(0.013)\end{array}$ & $\begin{array}{c}0.747 \\
(0.011)\end{array}$ & $\begin{array}{c}0.782 \\
(0.011)\end{array}$ & $\begin{array}{c}0.758 \\
(0.012)\end{array}$ & $\begin{array}{c}0.758 \\
(0.011)\end{array}$ & $\begin{array}{c}0.764 \\
(0.005)\end{array}$ \\
\hline \multicolumn{8}{|c|}{$A m y+($ slow $) 2$} \\
\hline 23 May & $\begin{array}{l}0.771 \\
(0.061)\end{array}$ & - & $\begin{array}{c}0.860 \\
(0.025)\end{array}$ & $\begin{array}{c}0.771 \\
(0.031)\end{array}$ & - & $\begin{array}{c}0.805 \\
(0.028)\end{array}$ & $\begin{array}{c}0.810 \\
(0.016)\end{array}$ \\
\hline 6 June & $\begin{array}{l}0.833 \\
(0.036)\end{array}$ & - & $\begin{array}{c}0.820 \\
(0.027)\end{array}$ & $\begin{array}{c}0.799 \\
(0.035)\end{array}$ & $\begin{array}{c}0.845 \\
(0.026)\end{array}$ & $\begin{array}{c}0.730 \\
(0.031)\end{array}$ & $\begin{array}{c}0.803 \\
(0.014)\end{array}$ \\
\hline 20 June & $\begin{array}{l}0.808 \\
(0.045)\end{array}$ & $\begin{array}{c}0.840 \\
(0.026)\end{array}$ & $\begin{array}{c}0.820 \\
(0.027)\end{array}$ & $\begin{array}{c}0.810 \\
(0.028)\end{array}$ & $\begin{array}{c}0.825 \\
(0.027)\end{array}$ & $\begin{array}{c}0.845 \\
(0.026)\end{array}$ & $\begin{array}{c}0.827 \\
(0.012)\end{array}$ \\
\hline 11 July & $\begin{array}{l}0.795 \\
(0.029)\end{array}$ & $\begin{array}{c}0.805 \\
(0.028)\end{array}$ & $\begin{array}{c}0.835 \\
(0.026)\end{array}$ & $\begin{array}{c}0.850 \\
(0.025)\end{array}$ & $\begin{array}{c}0.860 \\
(0.025)\end{array}$ & $\begin{array}{c}0.848 \\
(0.026)\end{array}$ & $\begin{array}{c}0.832 \\
(0.011)\end{array}$ \\
\hline 1 August & $\begin{array}{l}0.840 \\
(0.026)\end{array}$ & $\begin{array}{c}0.845 \\
(0.026)\end{array}$ & $\begin{array}{c}0.870 \\
(0.024)\end{array}$ & $\begin{array}{c}0.825 \\
(0.027)\end{array}$ & $\begin{array}{c}0.845 \\
(0.026)\end{array}$ & $\begin{array}{c}0.830 \\
(0.027)\end{array}$ & $\begin{array}{c}0.843 \\
(0.011)\end{array}$ \\
\hline 12 September & $\begin{array}{l}0.844 \\
(0.027)\end{array}$ & $\begin{array}{c}0.837 \\
(0.026)\end{array}$ & $\begin{array}{c}0.790 \\
(0.029)\end{array}$ & $\begin{array}{c}0.846 \\
(0.031)\end{array}$ & $\begin{array}{c}0.785 \\
(0.029)\end{array}$ & $\begin{array}{c}0.850 \\
(0.036)\end{array}$ & $\begin{array}{c}0.821 \\
(0.012)\end{array}$ \\
\hline 10 October & $\begin{array}{l}0.865 \\
(0.024)\end{array}$ & $\begin{array}{c}0.880 \\
(0.023)\end{array}$ & $\begin{array}{c}0.897 \\
(0.022)\end{array}$ & $\begin{array}{c}0.846 \\
(0.026)\end{array}$ & $\begin{array}{c}0.822 \\
(0.029)\end{array}$ & $\begin{array}{c}0.830 \\
(0.027)\end{array}$ & $\begin{array}{c}0.857 \\
(0.010)\end{array}$ \\
\hline
\end{tabular}


Table 3 (Continued)

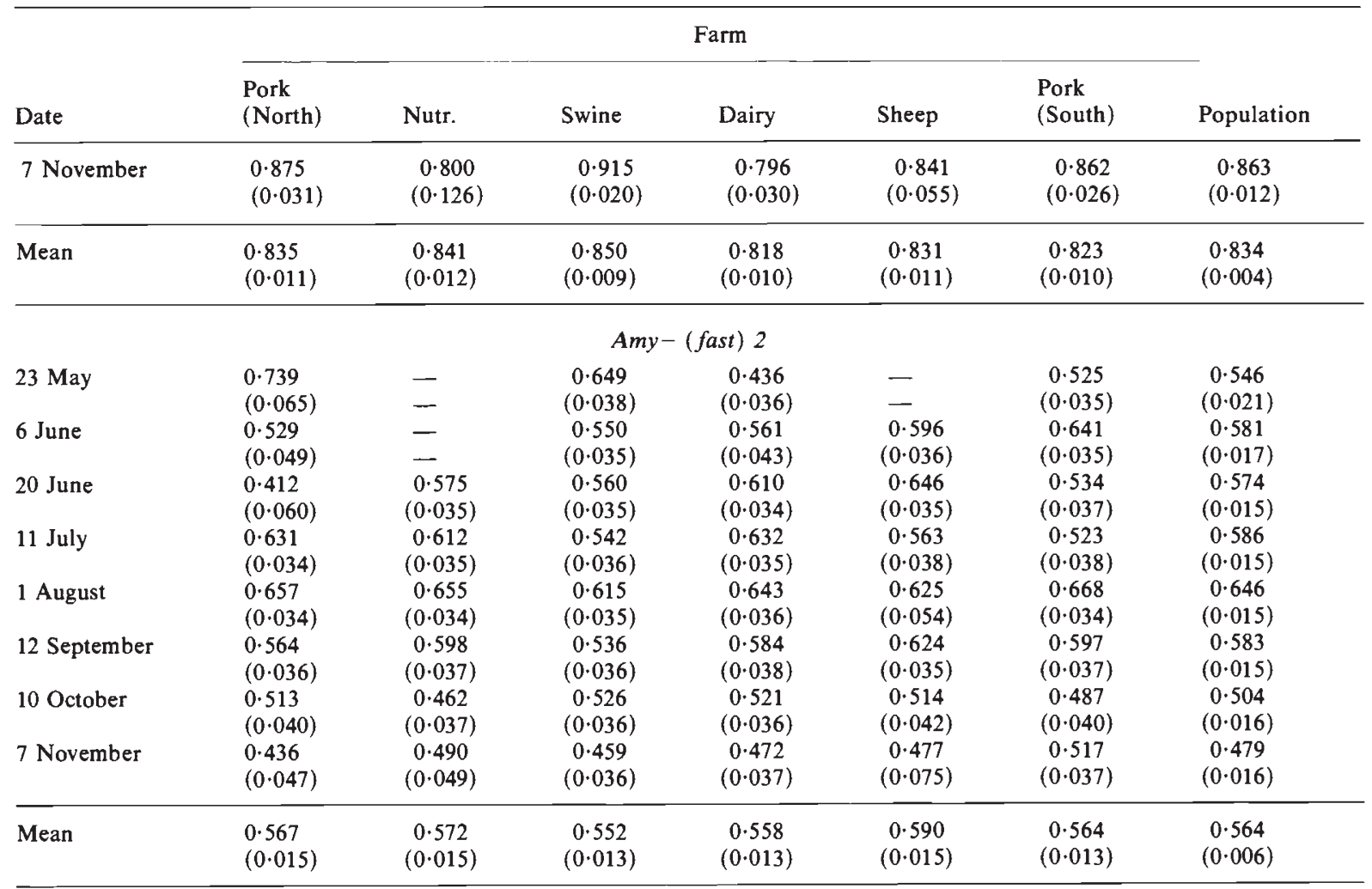

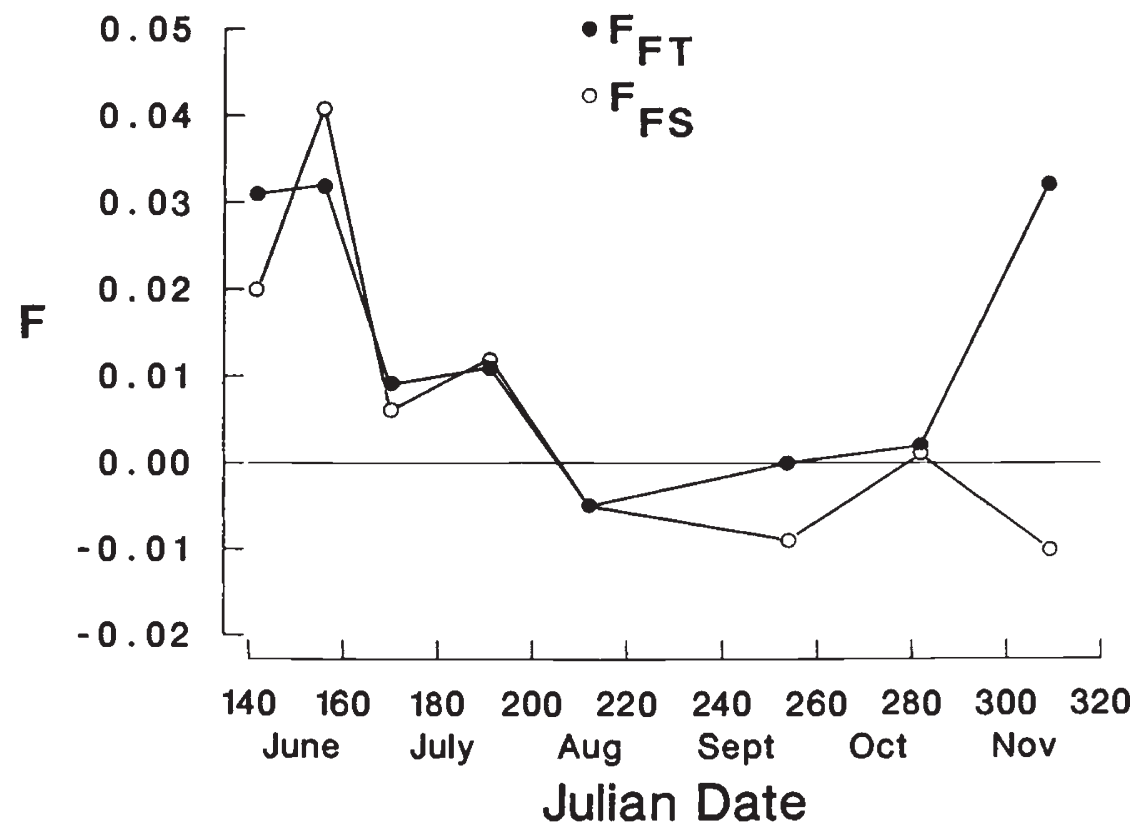

Figure 3 Seasonal fluctuations in Wright's $F_{\mathrm{FT}}$ and $F_{\mathrm{FS}}$ among parous flies. 


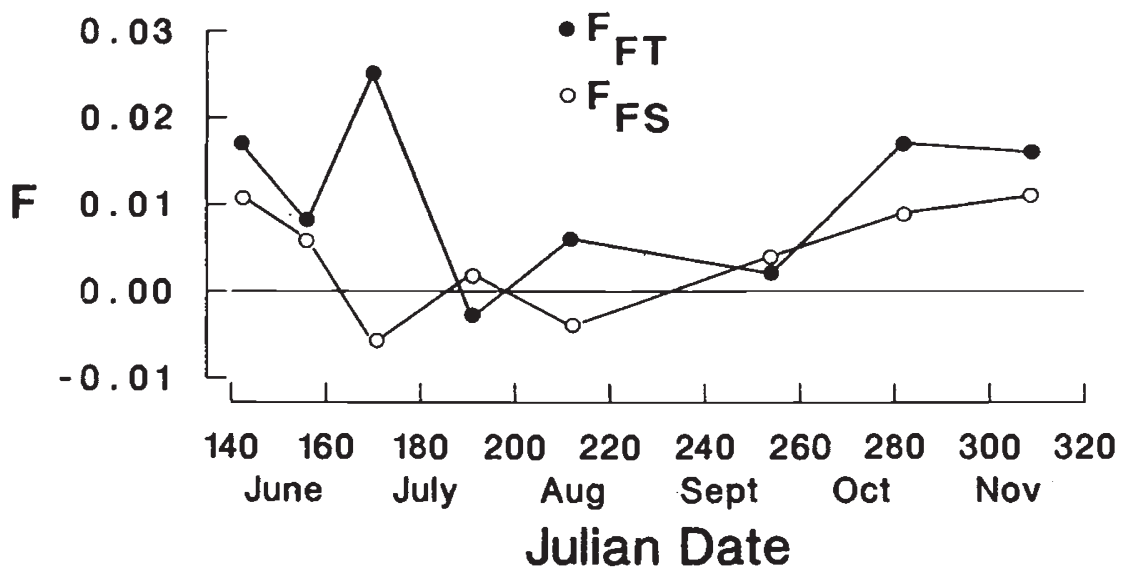

Figure 4 Seasonal fluctuations in Wright's $F_{\mathrm{Fr}}$ and $F_{\mathrm{FS}}$ among “young" flies.

Table 4 Correlation coefficients between allele frequencies at six farms

\begin{tabular}{|c|c|c|c|c|}
\hline \multirow[b]{2}{*}{ Subpopulations } & \multicolumn{4}{|c|}{ Allele } \\
\hline & Adh 2 & $\begin{array}{l}\text { Amy+ } \\
\text { (fast) } 4\end{array}$ & $\begin{array}{l}\text { Amy+ } \\
\text { (slow) } 2\end{array}$ & Amy -2 \\
\hline Nutrition $\times$ swine & 0.06 & $0 \cdot 13$ & $0 \cdot 12$ & $0 \cdot 78$ \\
\hline Nutrition $\times$ dairy & 0.61 & $-0 \cdot 30$ & $0 \cdot 36$ & $0.91^{*}$ \\
\hline Nutrition $\times$ sheep & 0.47 & 0.55 & -0.42 & $0 \cdot 78$ \\
\hline Nutrition $\times$ pork (south) & 0.64 & -0.02 & $-0.82^{*}$ & $0 \cdot 80$ \\
\hline Nutrition $\times$ pork (north) & $0 \cdot 89^{*}$ & $-0 \cdot 29$ & $0 \cdot 24$ & $0.67^{\circ}$ \\
\hline Swine $\times$ dairy & $0 \cdot 81^{*}$ & -0.45 & $-0 \cdot 18$ & 0.02 \\
\hline Swine $\times$ sheep & 0.60 & $-0 \cdot 35$ & 0.40 & $0 \cdot 78^{*}$ \\
\hline Swine $\times$ pork (south) & 0.60 & $-0 \cdot 27$ & $0 \cdot 20$ & $0 \cdot 32$ \\
\hline Swine $\times$ pork (north) & 0.42 & 0.00 & $0 \cdot 38$ & $0.77^{*}$ \\
\hline Dairy $\times$ sheep & 0.61 & -0.60 & $-0 \cdot 29$ & $0 \cdot 80^{*}$ \\
\hline Diary $\times$ pork (south) & 0.59 & $0 \cdot 15$ & 0.40 & 0.49 \\
\hline Dairy $\times$ pork (north) & $0 \cdot 72^{*}$ & 0.44 & $0 \cdot 31$ & -0.06 \\
\hline Sheep $\times$ pork (south) & $0 \cdot 15$ & 0.38 & $-0 \cdot 24$ & 0.62 \\
\hline Sheep $\times$ pork $($ north $)$ & $0 \cdot 40$ & 0.04 & $-0 \cdot 30$ & $0 \cdot 25$ \\
\hline Pork (south) $\times$ pork (north) & 0.64 & 0.67 & $0 \cdot 18$ & $0 \cdot 23$ \\
\hline Common correlation & 0.59 & 0.02 & 0.04 & 0.57 \\
\hline
\end{tabular}

*P $\leqq 0.05$

\section{DISCUSSION}

Seasonal breeding structure differed among old and young adult flies. Flies in both age groups, however, were heterogeneous at farms in spring because of drift. But older flies became panmictic in July and remained so until November. At that time subpopulations again became differentiated. Young flies remained differentiated throughout the season, and on only two sampling occasions were allele frequencies homogeneous. In autumn, young flies at farms became increasingly differentiated, and a large proportion of this variation was accounted for by drift.
Table 5 Frequencies and standard errors (in parentheses) of the most common allele at four enzymatic loci in overwintering populations of house flies

\begin{tabular}{|c|c|c|c|c|}
\hline \multirow[b]{2}{*}{$\begin{array}{l}\text { Date and } \\
\text { location }\end{array}$} & \multicolumn{4}{|c|}{ Allele } \\
\hline & $\operatorname{Adh} 2$ & $\begin{array}{l}\text { Amyt } \\
\text { (fast) } 4\end{array}$ & $\begin{array}{l}\text { Amyt } \\
\text { (slow) } 2\end{array}$ & $A m y-2$ \\
\hline \multicolumn{5}{|c|}{10 October 1982} \\
\hline Swine & $\begin{array}{c}0.750 \\
(0.043)\end{array}$ & $\begin{array}{c}0.830 \\
(0.038)\end{array}$ & - & - \\
\hline Population & $\begin{array}{c}0.711 \\
(0.020)\end{array}$ & $\begin{array}{c}0.746 \\
(0.020)\end{array}$ & - & - \\
\hline \multicolumn{5}{|c|}{23 January 1983} \\
\hline Swine (brick) & $\begin{array}{c}0.806 \\
(0.047)\end{array}$ & $\begin{array}{c}0.833 \\
(0.044)\end{array}$ & $\begin{array}{c}0.833 \\
(0.044)\end{array}$ & $\begin{array}{c}0.829 \\
(0.045)\end{array}$ \\
\hline Swine (wood) & $\begin{array}{l}0.647 \\
(0.058)\end{array}$ & $\begin{array}{c}0.750 \\
(0.053)\end{array}$ & $\begin{array}{c}0.824 \\
(0.046)\end{array}$ & $\begin{array}{c}0.676 \\
(0.057)\end{array}$ \\
\hline Mean & $\begin{array}{c}0.729 \\
(0.038)\end{array}$ & $\begin{array}{c}0.793 \\
(0.034)\end{array}$ & $\begin{array}{c}0.829 \\
(0.032)\end{array}$ & $\begin{array}{c}0.754 \\
(0.037)\end{array}$ \\
\hline \multicolumn{5}{|l|}{10 April 1983} \\
\hline Swine (brick) & $\begin{array}{c}0.686 \\
(0.043)\end{array}$ & $\begin{array}{c}0.780 \\
(0.038)\end{array}$ & $\begin{array}{c}0.856 \\
(0.032)\end{array}$ & $\begin{array}{c}0.746 \\
(0.040)\end{array}$ \\
\hline Swine (wood) & $\begin{array}{c}0.659 \\
(0.052)\end{array}$ & $\begin{array}{c}0.695 \\
(0.051)\end{array}$ & $\begin{array}{c}0.817 \\
(0.043)\end{array}$ & $\begin{array}{c}0.720 \\
(0.050)\end{array}$ \\
\hline Mean & $\begin{array}{c}0.675 \\
(0.033)\end{array}$ & $\begin{array}{c}0.745 \\
(0.031)\end{array}$ & $\begin{array}{c}0.840 \\
(0.026)\end{array}$ & $\begin{array}{c}0.735 \\
(0.031)\end{array}$ \\
\hline
\end{tabular}

Seasonal trends in breeding structure among "young" flies were similar in 1982 and 1983. In each year, young flies at farms became differentiated in August when populations were panmictic. This establishes the hypothesis that the differentiation resulted from larval adaptation to local conditions. Heterogeneity was detected because the frequency of migration in the youngest adult age group was trivial. Allele frequencies in young flies therefore reflected the habitat in which they matured. 
In the winter of 1982-1983, drift did not differentiate flies in the two adjacent farrowing sheds at the swine farm. In November 1983 allele frequencies in older flies were heterogeneous among, but homogeneous within, subpopulations. Thus, subpopulations drifted apart in autumn, but overwintering colonies at nearby farms were homogeneous. As winter progressed, frequencies at farms drifted from the initial values in the founding colonies, and by spring, gene frequencies at adjacent farms also had drifted. Allele frequencies in the general population, however, were unchanged during winter. This result is not surprising because of the large number of livestock facilities in Iowa where house fly populations can overwinter. Also, generation time in winter became greatly extended because temperatures were low. Generations overlap continuously, but only two or three generations probably obtain between November and April.

The data suggest a cyclical pattern of breeding structure in house flies. In autumn, subpopulations become differentiated by genetic drift. Actively reproducing overwintering populations become established in heated domestic livestock facilities. Founding populations are sufficiently large to prevent inbreeding or immediate genetic drift. Allele frequencies at farms gradually drift from frequencies in founding colonies so that, by spring, flies at adjacent farms are differentiated. Populations exponentially increase in June, and differentiation declines rapidly. Flies become detectably panmictic in July and remain so until October or November, when they again drift apart. The differentiation at farms observed among young adults reflects larval adaptation to the different resources. House fly females become inseminated early, at about the time vitellogenesis begins, and their most likely mates are young, locally reared males (Krafsur et al., 1985). The first oviposition, moreover, is also likely to take place locally as is shown by the genetic structure of the age graded samples. But the resulting population differentiation is dissipated by immigration of parous adults and the occassional mating of a local female by an immigrant male.

Temporal fluctuations in the frequencies of $A d h$ (van Delden, 1982) and Amy (Doane, 1980) genes are well documented in Drosophila species. Kinetic differences have been reported among allozymes at both loci and allele frequencies have been correlated with a variety of environmental factors. Among house fly larvae, $A d h$ may be an important detoxifying enzyme because they breed in fermenting substrates. The house fly may prove to be a valuable species for research on the adaptive significance of amylase polymorphisms. Only a single amylase locus is known in Drosophila, but we have identified six amylase loci in the house fly, one of which is very active and transcribed only in larvae. Studies on the kinetics of amylase allozymes might answer interesting questions raised in the current study. Why is there a larval amylase? Why does selection seem to act on Amy - and not the other Amy loci? The house fly is easily studied in the field or laboratory and thus presents many new opportunities for population geneticists interested in the evolutionary consequences of breeding structure and the functional significance of enzyme polymorphisms.

\section{REFERENCES}

BISHOPP, F. C. 1921. Dispersion of flies by flight. J. Agric. Res. (Wash.), 21, 729-766.

BLACK, W. C. AND KRAFSUR, E. S. 1984. Vertical slab gel electrophoresis on a large number of samples: An apparatus with the capacity for central cooling. Anal. Bio chem., 138, 210-216.

BLACK, W. C. AND KRAFSUR, E. S. 1985a. Electrophoretic analysis of genetic variability in the house fly (Musca domestica L.). Biochem. Genet., 23, 191-201.

BLACK, W. C. AND KRAFSUR, E. S. 1985b. A FORTRAN program for analysis of genotypic frequencies and description of the breeding structure of populations. Theor. Appl. Genet., 70, 484-490.

BLACK, W. C. AND KRAFSUR, E. S. 1985c. A FORTRAN program for the calculation and analysis of two-locus linkage disequilibrium coefficients. Theor. Appl. Genet., 70, 491496.

BLACK, W. C. AND KRAFSUR, E. S. 1986a. Population biology and genetics of winter house fly (Diptera: Muscidae) populations. Ann. Entomol. Soc. Am. (in press).

BLACK, W. C. AND KRAFSUR, E. S. 1986b. Temporal and spatial trends in allozyme frequencies in house fly populations, Musca domestica L. Theor. Appl. Genet., 71, 673-681.

DOANE, w. W. 1980. Selection for amylase allozymes in Drosophila melanogaster: Some questions. Evolution, 34, 868-874.

EANES, W. F. AND KOEHN, R. K. 1978. An analysis of genetic structure in the monarch butterfly, Danaus plexippus L. Evolution, 32, 787-797.

ELVIN, M. K. AND KRAFSUR, E. S. 1984. Relationship between temperature and rate of ovarian development in the house fly, Musca domestica L. (Diptera: Muscidae) Ann. Entomol. Soc. Am., 77, 50-55.

HARTL, D. L. 1980. Principles of population genetics. Sinauer Assoc., Inc. Sunderland, Mass.

JOHNSON, M. S. 1976. Allozymes and area effects in Cepaea nemoralis on the western Berkshire downs. Heredity, 36, 105-121.

KRAFSUR, E. S., BLACK, W. C., CHURCH, C. J., AND BARNES, D. A. 1985. Age structure and reproductive biology of a house fly (Diptera: Muscidae) population. Environ. Entomol., 14, 159-164. 
KRAFSUR, E. S. 1985. Age composition and seasonal phenology of house fly (Diptera: Muscidae) populations. J. Med. Entomol., 22, 515-523.

SELANDER, R. K. AND KAUFMANN, D. W. 1975. Genetic structure of populations of the brown snail (Helix aspersa): I. Microgeographic variation. Evolution, 29, 385-401.

SOKAL, R. R. AND ROHLF, F. J. 1969. Biometry. W. H. Freeman \& Co., San Francisco.

VAN DELDEN, w. 1982. The alcohol dehydrogenase polymorphism in Drosophila melanogaster: Selection at an enzyme locus. Evol. Biol., 15, 187-222.

WEIR, B. S. AND COCKERHAM, C. C. 1985. Estimating F-statistics for the analysis of population structure. Evolution, 38, $1350-1370$.
WORKMAN, P. L. AND NISWANDER, J. D. 1970. Population studies on southwestern Indian tribes. II. Local genetic differentiation in the Papago. Am. J. Human Genét, 22, 24-49.

WRIGHT, S. 1951. The genetical structure of populations. Ann. Eugenics, 15, 323-354.

WRIGHT, S. 1965. The interpretation of population structure by $F$-statistics with special regard to systems of mating. Evolution, 19, 395-420.

WRIGHT, S. 1978. Evolution and the genetics of Populations. Vol. 4, Variability within and among natural populations. University of Chicago Press, Chicago. 